\title{
Web Usage Mining: Extracting Unexpected Periods from Web Logs
}

\author{
F. Masseglia ${ }^{(1)}$ \\ P. Poncelet ${ }^{(2)}$ \\ M. Teisseire ${ }^{(3)}$ \\ A. Marascu ${ }^{(1)}$ \\ ${ }^{(1)}$ INRIA Sophia Antipolis - AxIS Project/Team, 2004 route des Lucioles - BP 93, 06902 Sophia Antipolis, \\ France \\ email: \{Florent.Masseglia, Alice.Marascu\}@sophia.inria.fr \\ (2)EMA-LGI2P/Site EERIE, Parc Scientifique Georges Besse, 30035 Nîmes Cedex 1 \\ email: Pascal.Poncelet@ema.fr \\ ${ }^{(3)}$ LIRMM UMR CNRS 5506, 161 Rue Ada, 34392 Montpellier Cedex 5 \\ email: teisseire@lirmm.fr
}

\begin{abstract}
Existing Web Usage Mining techniques are currently based on an arbitrary division of the data (e.g. "one log per month") or guided by presumed results (e.g "what is the customers behaviour for the period of Christmas purchases?"). Those approaches have two main drawbacks. First, they depend on this arbitrary organization of the data. Second, they cannot automatically extract "seasons peaks" among the stored data. In this paper, we propose to perform a specific data mining process (and particularly to extract frequent behaviours) in order to automatically discover the densest periods. Our method extracts, among the whole set of possible combinations, the frequent sequential patterns related to the extracted periods. A period will be considered as dense if it contains at least one frequent sequential pattern for the set of users connected to the Web site in that period. Our experiments show that the extracted periods are relevant and our approach is able to extract both frequent sequential patterns and the associated dense periods.
\end{abstract}

\section{Introduction}

Analyzing the behaviour of a Web Site users, also known as Web Usage Mining, is a research field which consists in adapting the data mining methods to access log files records. These files collect data such as the IP address of the connected machine, the requested URL, the date and other information regarding the navigation of the user. Web Usage Mining techniques provide knowledge about the behaviour of the users in order to extract relationships in the recorded data $[5,15,19]$. Among available techniques, the sequential patterns [2] are particularly well adapted to the log study. Extracting sequential patterns on a $\log$ file, is supposed to provide the following kind of relationship: "On the INRIA's Web Site, $10 \%$ of users visited consecutively the homepage, the available positions page, the ET $T^{1}$ offers, the ET missions and finally the past ET competitive selection".

This kind of behaviour is only supposed to exist, because extracting sequential patterns on a log file means to manage several problems, such as:

- Caches (on the client machine) and proxies (acting as local caches) which lower the number of records in the log file (e.g. parts of a navigation that will be kept in a cache and thus never recorded in the $\log$ file).

${ }^{1}$ ET: Engineers, Technicians. 
- Great diversity of pages on the site. For instance, on a Web site such as Inria's, there are up to 70,000 filtered resources (after the data selection step of KDD) for the main site and 82,0000 resources for the site of Sophia Antipolis.

- Search engines which allow the user to directly access a specific part of the Web site. This may lower the number of records in the log file and the number of navigations shared by different users (i.e. the potential common prefix of navigations) if they don't search for the resources through a site map for instance.

In this paper, we will focus on a particular problem that has to be considered by Web Usage Mining techniques: the arbitrary division of the data which is done today. This division comes either from an arbitrary decision in order to provide one log per $x$ days (e.g. one log per month), or from a wish to find particular behaviours (e.g. the behaviour of the Web site users from November 15 to December 23, during Christmas purchases). In order to better understand our goal, let us consider student behaviours when they are connected for a working session. Let us assume that these students belong to two different groups having twenty students. The first group was connected on 31/01/05 while the other one was connected on $01 / 02 / 05$, (i.e. the second group was connected one day later). During the working session, students have to perform the following navigation: First they access URL "www-sop.inria.fr/cr/tp_accueil.html", then "www-sop.inria.fr/cr/tp1_accueil.html" which will be followed by "www-sop.inria. fr/cr/tp1a.html".

Let us consider, as it is usual in traditional approaches, that we analyze access logs per month. During January, we only can extract twenty similar behaviours, among 200,000 navigations on the log, sharing the working session. Furthermore, even when considering a range of one month or of one year, this sequence of navigation does not appear sufficiently on the logs $(20 / 20000)$ and will not be easy to extract. Let us now consider that we are provided with logs for a very long period (e.g. several years). With the method developed in this article, we can find that it exists at least one dense period in the range [31/01-01/02]. Furthermore, we know that, during this period, 340 users were connected. We are thus provided with the new following knowledge: $11 \%$ (i.e. 40 on 340 connected users) of users visited consecutively the URLs "tp_accueil.html", "tp1_accueil.html", and finally "tp1a.html".

Efficient tools are proposed today [22, 10] for analyzing logs at different level of granularity (day, month, year). They allow for instance to know how many time the site is accessed or how many requests have been done on each page. Nevertheless, as they depend on the chosen granularity, they suffer the previously addressed drawback: they cannot obtain frequent patterns on a very short period because usually such patterns do not appear sufficiently on the whole log. Close to our problem, [14] propose to extract episodes rules on a long sequence as well as the optimal window size. Nevertheless our problem is very different since we do not consider that we are provided with a unique long sequence. In our context, i.e. access logs, sequences correspond to different behaviours of users on a Web Server. Then we have to manage a very huge set of data sequences and we have to extract both frequent sequences and the period where these sequences appear.

The remainder of this paper is organized as follows. Section 2 goes deeper into presenting sequential patterns and how they can be used on Web Usage Mining. In Section 3, we give an overview of Web Usage Mining approaches which are based on sequential patterns. Section 4 presents our motivation for a new approach. Our solution based on a new heuristic called PERIO is presented in Section 5. Experiments are reported Section 6, and Section 7 concludes the paper with future avenues for research.

\section{Definitions}

In this section we define the sequential pattern mining problem in large databases and give an illustration. Then we explain the goals and techniques of Web Usage Mining with sequential patterns. 
The sequential pattern mining definitions are those given by [1] and [20].

\subsection{Sequential pattern mining}

In [1], the association rules mining problem is defined as follows:

Definition 1 Let $I=\left\{i_{1}, i_{2}, \ldots, i_{m}\right\}$, be a set of $m$ literals (items). Let $D=\left\{t_{1}, t_{2}, \ldots t_{n}\right\}$, be a set of $n$ transactions; Associated with each transaction is a unique identifier called its TID and an itemset I. $I$ is a $k$-itemset where $k$ is the number of items in $I$. We say that a transaction $T$ contains $X$, a set of some items in $I$, if $X \subseteq T$. The support of an itemset $I$ is the fraction of transactions in $D$ containing $I: \operatorname{supp}(I)=\|\{t \in D \mid I \subseteq t\}\| /\|\{t \in D\}\|$. An association rule is an implication of the form $I_{1} \Rightarrow I_{2}$, where $I_{1}, I_{2} \subset I$ and $I_{1} \cap I_{2}=\emptyset$. The rule $I_{1} \Rightarrow I_{2}$ holds in the transaction set $D$ with confidence $c$ if $c \%$ of transactions in $D$ that contain $I_{1}$ also contain $I_{2}$. The rule $r: I_{1} \Rightarrow I_{2}$ has support $s$ in the transaction set $D$ if $s \%$ of transactions in $D$ contain $I_{1} \cup I_{2}$ (i.e. $\operatorname{supp}(r)=\operatorname{supp}\left(I_{1} \cup I_{2}\right)$ ).

Given two parameters specified by the user, minsupp and minconfidence, the problem of association rule mining in a database $D$ aims at providing the set of frequent itemsets in $D$, i.e. all the itemsets having support greater or equal to minsupp. Association rules with confidence greater than minconfidence are thus generated.

As this definition does not take time into consideration, the sequential patterns are defined in [20]:

Definition 2 A sequence is an ordered list of itemsets denoted by $\left\langle s_{1} s_{2} \ldots s_{n}>\right.$ where $s_{j}$ is an itemset. The data-sequence of a customer $c$ is the sequence in $D$ corresponding to customer $c$. A sequence $\left\langle a_{1} a_{2} \ldots a_{n}\right\rangle$ is a subsequence of another sequence $\left\langle b_{1} b_{2} \ldots b_{m}\right\rangle$ if there exist integers $i_{1}<i_{2}<\ldots<i_{n}$ such that $a_{1} \subseteq b_{i_{1}}, a_{2} \subseteq b_{i_{2}}, \ldots, a_{n} \subseteq b_{i_{n}}$.

Example 1 Let $C$ be a client and $S=<(3)$ (4 5) (8) >, be that client's purchases. $S$ means that " $C$ bought item 3, then he or she bought 4 and 5 at the same moment (i.e. in the same transaction) and finally bought item 8".

Definition 3 The support for a sequence $s$, also called $\operatorname{supp}(s)$, is defined as the fraction of total data-sequences that contain $s$. If $\operatorname{supp}(s) \geq$ minsupp, with a minimum support value minsupp given by the user, $s$ is considered as a frequent sequential pattern.

\subsection{Access log files analysis with sequential patterns}

The general idea is similar to the principle proposed in [7]. It relies on three main steps. First of all, starting from a rough data file, a pre-processing step is necessary to clean "useless" information. The second step starts from this pre-processed data and applies data mining algorithms to find frequent itemsets or frequent sequential patterns. Finally, the third step aims at helping the user to analyze the results by providing a visualization and request tool.

Raw data is collected in access log files by Web servers. Each input in the log file illustrates a request from a client machine to the server ( $h t t p$ daemon). Access log files format can differ, depending on the system hosting the Web site. For the rest of this presentation we will focus on three fields: client address, the URL asked for by the user and the time and date for that request. We illustrate these concepts with the access log file format given by the CERN and the NCSA [4], where a log input contains records made of 7 fields, separated by spaces [17]:host user authuser [date:time] "request" status bytes

The access $\log$ file is then processed in two steps. First of all, the access log file is sorted by address and by transaction. Then each "uninteresting" data is pruned out from the file. During the sorting process, in order to allow the knowledge discovery process to be more efficient, URLs and clients are mapped into integers. Each time and date is also translated into relative time, compared to the earliest time in the log file. 


\begin{tabular}{|c|c|c|c|c|c|}
\hline Client & $\mathrm{d} 1$ & $\mathrm{~d} 2$ & $\mathrm{~d} 3$ & $\mathrm{~d} 4$ & $\mathrm{~d} 5$ \\
\hline 1 & 10 & 30 & 40 & 20 & 30 \\
\hline 2 & 10 & 30 & 20 & 60 & 30 \\
\hline 3 & 10 & 70 & 30 & 20 & 30 \\
\hline
\end{tabular}

Table 1: File obtained after a pre-processing step

Definition 4 Let $\log$ be a set of server access log entries.

An entry $g, g \in L o g$, is a tuple $g=<i p_{g},\left(\left[l_{1}^{g} . U R L, l_{1}^{g}\right.\right.$. time $] \ldots\left[l_{m}^{g} . U R L, l_{m}^{g}\right.$. time $\left.]\right)>$ such that for $1 \leq k \leq m, l_{k}^{g} . U R L$ is the item asked for by the user $g$ at time $l_{k}^{g}$.time and for all $1 \leq j<k, l_{k}^{g}$.time $>$ $l_{j}^{g}$. time.

The structure of a log file, as described in Definition 4, is close to the "Client-Time-Item" structure used by sequential pattern algorithms. In order to extract frequent behavior from a log file, for each $g$ in the log file, we first have to transform $i p_{g}$ into a client number and for each record $k$ in $g$, $l_{k}^{g}$.time is transformed into a time number and $l_{k}^{g} . U R L$ is transformed into an item number. Table 1 gives a file example obtained after that pre-processing. To each client corresponds a series of times and the URL requested by the client at each time. For instance, the client 2 requested the URL "60" at time $d 4$.

The goal is thus, according to definition 3 and by means of a data mining step, to find the sequential patterns in the file that can be considered as frequent. The result may, for instance be $\langle(10)(30)(20)(30)\rangle$ (with the file illustrated in figure 1 and a minimum support given by the user: 100\%). Such a result, once mapped back into URLs, strengthens the discovery of a frequent behavior, common to $n$ users (with $n$ the threshold given for the data mining process) and also gives the sequence of events composing that behavior.

\section{Related Work}

Several techniques for extracting sequential patterns have been applied to access log files $[13,19,3,9$, $23,16]$. Sequential patterns can have some qualities compared to association rules, such as the notion of time embedded in the extracted knowledge. The interested reader may refer to [16] for a detailed comparison between association rules and sequential patterns for Web Usage Mining.

In this section, we describe three main set of studies that use the notion of sequences for Web Usage Mining.

First applications of sequential patterns for Web usage analysis:

The WUM (Web Utilisation Miner) tool proposed by [19] allows discovering navigation patterns that will be interesting from the statistical point of view. The pattern extraction proposed in WUM relies on the frequency (minimum support) of the considered patterns. It is also possible to specify another criteria for the patterns such as some URLs that have to be included in the navigation or the confidence between two or more pages of the navigation pattern.

In [13], the authors propose the WebTool system. In this system, sequential pattern mining relies on PSP, an algorithm developed by the authors. The originality consists in the prefix tree designed to manage at the same time the candidates and the frequent patterns.

Extraction techniques similar to sequential pattern mining methods:

In [9] the authors propose to consider the notion of time embedded in the navigations for clustering the user sessions. This clustering relies on an alignment algorithm in order to measure their distance. 
The main contribution is a study of the clusters quality, by comparison (in their experimentations) to the clusters obtained thanks to a distance based on the itemsets (without the notion of time).

The authors of [23] consider the navigations of a Web site's users as a Markov chain. The paper is mainly about problems related to Markov models and the transition matrix computed for each log. The authors propose a compression algorithm of the transition matrix in order to obtain an efficient link prediction.

Quality of the results and characteristics of the site:

More recently, studies about Web usage analysis have focused on the quality of the results, their relevance and their utility. This is also the case for works based on the notion of sequence.

In [16] the authors show that the characteristics of the site have an impact on the quality of the modifications proposed to the users depending on whether those propositions are based on frequent itemsets or frequent sequences (such as sequential patterns). Three main characteristics are considered: the topology, the degree of connectivity and the length of potential navigations. The patterns are first extracted from the first part of the log. For each extraction method the patterns are used to evaluate the relevance of the predictions that would be made. That relevance is evaluated on the second part of the log. Their experiments show the relevance of itemsets for the sites having a high degree of connectivity. On the other hand, they show the relevance of sequential patterns for the sites having long potential navigations (including the sites with dynamic content).

The methods presented in this section, such as (to the best of our knowledge) any existing method for mining Web logs, depend on an arbitrary division of the data. In the next section, we propose to understand the goal of our study and its general principle.

\section{Motivation and General Principle}

This section is devoted to motivating our proposal regarding the relevance and utility of the tackled knowledge. It also illustrates the issues involved and the general principle of our method.

\subsection{Motivation}

The outline of our method is the following: enumerating the sets of periods in the log that will be analyzed and then identifying which ones contain frequent sequential patterns. In this section we will define the notions of period and frequent sequential patterns over a period. Let us consider the set of transactions in Figure 1 (upper left table). Those transactions are sorted by timestamp, as they would be in a $\log$ file. In this table containing 9 records, the customer $c_{1}$, for instance, has connected at time 1 and requested the URL $a$. Let us now consider the "in" and "out" timestamps of each client, reporting their arrival and departure (upper right table in Figure 1). The first request of client $c_{1}$ has occurred at time 1 , and the last one at time 4 . We can thus report the periods of that log. In the example of Figure 1 there are 5 periods. During the first period (from time 1 to time 2), the client $c_{1}$ was the only one connected on the Web site. Then, clients $c_{1}$ and $c_{2}$ are connected during the same period $p_{2}$ (from time 3 to time 4), and so on.

Let us now consider the navigation sequences of the log represented in Figure 1. Those sequences are reported in Figure 2, as well as the frequent sequential patterns extracted on the whole log and on the identified periods. With a minimum support of $100 \%$ on the whole $\log$, the only frequent sequential pattern is merely reduced to the item $d:\langle(d)\rangle$. Let us now consider the periods identified above, as well as the customers connected for each period. For the periods $p_{1}, p_{3}$ and $p_{5}$, reduced to a single client, there is no relevant frequent pattern. For the period $p_{2}$ a sequential patterns is extracted: < 


\begin{tabular}{|c|c|c|}
\hline Cust_Id & time & URL \\
\hline$c_{1}$ & 1 & $a$ \\
\hline$c_{1}$ & 2 & $b$ \\
\hline$c_{2}$ & 3 & $a$ \\
\hline$c_{1}$ & 4 & $d$ \\
\hline$c_{2}$ & 5 & $d$ \\
\hline$c_{3}$ & 6 & $d$ \\
\hline$c_{2}$ & 7 & $e$ \\
\hline$c_{3}$ & 8 & $e$ \\
\hline$c_{3}$ & 9 & $f$ \\
\hline$c_{1}$ & 1 & 4 \\
\hline$c_{2}$ & 3 & 7 \\
\hline Cust_Id & In & Out \\
\hline$c_{3}$ & 6 & 9 \\
\hline
\end{tabular}

\begin{tabular}{|c|c|c|}
\hline Period & Begin/End & Customers \\
\hline$p_{1}$ & {$[1 . .2]$} & $c_{1}$ \\
\hline$p_{2}$ & {$[3 . .4]$} & $c_{1}, c_{2}$ \\
\hline$p_{3}$ & {$[5]$} & $c_{2}$ \\
\hline$p_{4}$ & {$[6 . .7]$} & $c_{2}, c_{3}$ \\
\hline$p_{5}$ & {$[8 . .9]$} & $c_{3}$ \\
\hline
\end{tabular}

Figure 1: A log containing three sequences and the associated periods

(a) $(d)>$. This pattern is common to both clients connected during the period $p_{2}: c_{1}$ and $c_{2}$. Finally, during period $p_{4}$, the pattern $<(d)(e)>$ is extracted.

\begin{tabular}{|c|c|c|c|c|c|}
\hline Cust_Id & Sequence & $\log$ & $p_{1}, p_{3}, p_{5}$ & $p_{2}$ & $p_{4}$ \\
\hline$c_{1}$ & $<(a)(b)(d)>$ & \multirow{3}{*}{$<(d)>$} & - & \multirow{3}{*}{$<(a)(d)>$} & \multirow{3}{*}{$<(d)(e)>$} \\
\hline$c_{2}$ & $<(a)(d)(e)>$ & & - & & \\
\hline$c_{3}$ & $<(d)(e)(f)>$ & & - & & \\
\hline
\end{tabular}

Figure 2: Frequent sequential patterns obtained for customers connected at each period

The following part of this section is devoted to more formal definitions of period, connected clients and stable periods. Let $C$ be the set of clients in the $\log$ and $D$ the set of recorded timestamps.

Definition $5 P$, the set of potential periods on the log is defined as follows:

$P=\left\{\left(p_{a}, p_{b}\right) /\left(p_{a}, p_{b}\right) \in D \times D\right.$ and $\left.a \leq b\right\}$.

In the following definition, we consider that $d_{\min }(c)$ and $d_{\max }(c)$ are respectively the arrival and departure time for $c$ in the $\log$ (first and last request recorded for $c$ ).

Definition 6 Let $C_{(a, b)}$ be the set of clients connected during the period $(a, b) . C_{(a, b)}$ is defined as follows:

$C_{(a, b)}=\{c / c \in C$ and $\left.\left[d_{\min }(c) . . d_{\max }(c)\right] \cap[a . . b] \neq \emptyset\right\}$.

Finally, we give the definitions of stable period and dense period. The first one is a maximal period $p_{m}$ during which $C_{p_{m}}$ does not vary. With the example given in Figure 1, the period [6..7] is a stable period. This is not the case for [3..3] which is included in [3..4] and contains the same clients (i.e. $\left.C_{(3,3)}=C_{(3,4)}\right)$. A dense period is a stable period containing at least a frequent sequential pattern. 
In the example given in section 1, the period corresponding to January 31 (i.e. during the working session) should be a dense period.

Definition 7 Let $P_{\text {stable }}$ be the set of stable periods. $P_{\text {stable }}$ is defined as follows:

$P_{\text {stable }}=\left\{\left(m_{a}, m_{b}\right) /\left(m_{a}, m_{b}\right) \in P\right.$ and

1) $\nexists\left(m_{a}^{\prime}, m_{b}^{\prime}\right) /(b-a)<\left(b^{\prime}-a^{\prime}\right)$

and $\left[a^{\prime} . . b^{\prime}\right] \cap[a . . b] \neq \emptyset$

and $C_{\left(m_{a}^{\prime}, m_{b}^{\prime}\right)}=C_{\left(m_{a}, m_{b}\right)}$

2) $\forall(x, y) \in[a . . b], \forall(z, t) \in[a . . b] /$

$x \leq y, z \leq t$ then $\left.C_{(x, y)}=C_{(z, t)} \cdot\right\}$

Condition 1 , in definition 7 , ensures that no largest period includes $\left(m_{a}, m_{b}\right)$ and contains the same clients. Condition 2 ensures that there is no arrival or departure inside any period of $P_{\text {stable }}$.

Definition 8 A stable period $p$ is dense if $C_{p}$ contains at least a frequent sequential pattern with respect to the minimum support specified by the user proportionally to $\left|C_{p}\right|$.

The notion of dense period (definition 8), is the core of this paper. In the following, our goal will be to extract those periods, as well as the corresponding frequent patterns, from the log file. In order to give an illustration, let us consider the period $p_{e}$ containing 100 clients $\left(\left|C_{p_{e}}\right|=100\right)$ and a minimum support of $5 \%$. Any sequential pattern included in at least 5 navigations of $C_{p_{e}}$ will be considered as frequent for that period. If there exists at least a frequent pattern in $p_{e}$ then this period has to be extracted by our method. Extracting the sequential patterns of each period by means of a traditional sequential pattern mining method is not a suitable solution for the following reasons. First, sequential pattern mining algorithms (such as PSP [12] or PrefixSpan [18] for instance) can fail if one of the patterns to extract is very long. When considering navigations on a Web site, it is usual to find numerous requests for a same URL (pdf or php files for instance). Finally, during our experiments, with a total amount of 14 months of $\log$ files, we detected approximately 3, 500, 000 stable periods. We believe that mining dense period by means of a heuristic is more relevant that several millions calls to a traditional algorithm for mining sequential patterns. The outline of our approach, intended to detect dense periods in the log file, is presented in the next section.

\subsection{General Principle}

Figure 3 gives an overview of the PERIO heuristic that we propose for solving the problem of dense period mining. First, starting from the log, the periods are detected. Those periods are then considered one by one and sorted by their "begin" timestamp. For each iteration $n$, the period $p_{n}$ is scanned. The set of clients $C_{p_{n}}$ is loaded in main memory (" $D B$ " in Figure 3). Candidates having length 2 are generated from the frequent items detected in $C_{p_{n}}$ (step "1" in Figure 3). Because of the large number of candidates generated, this operation only occurs every $s$ steps (where $s$ is a user defined parameter). Candidates are then compared to sequences of $C_{p_{n}}$ in order to detect the frequent patterns (step " 2 " in Figure 3). Frequent patterns are injected in the neighborhood operators described in Section 5.2.1 and the new generated candidates are compared with the sequences of $C_{p_{n}}$. In order to obtain a result as fine as possible on each period, it is possible for the user to give the minimum number of iteration $(j)$ on each period.

\subsection{Limits of Sequential Pattern Mining}

Our method will process the log file by considering millions of periods (each period corresponds to a sub$\log$ ). The principle of our method will be to extract frequent sequential patterns from each period. Let 


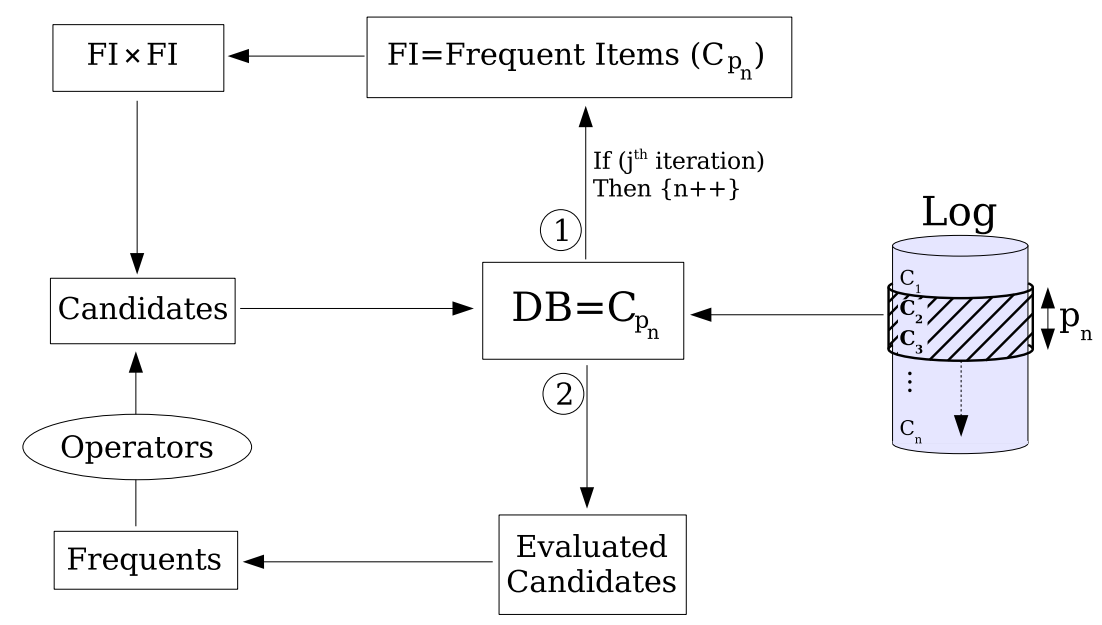

Figure 3: Overview of the operations performed by PERIO

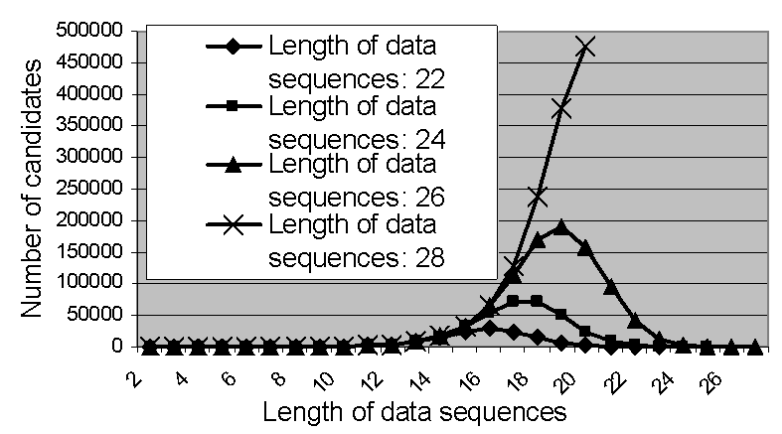

Figure 4: Limits of a framework involving PSP

us consider that the frequent sequences are extracted with a traditional exhaustive method (designed for a static transaction database). We argue that such a method will have at least one drawback leading to a blocking operator. Let us consider the example of the PSP [12] algorithm. We have tested this algorithm on databases containing only two sequences $\left(s_{1}\right.$ and $\left.s_{2}\right)$. Both sequences are equal and contain repetitions of itemsets having length one. The first database contains 11 repetitions of the itemsets $(1)(2)$ (i.e. $s_{1}=<(1)(2)(1)(2) \ldots(1)(2)>, \operatorname{lentgh}\left(s_{1}\right)=22$ and $s_{2}=s_{1}$ ). The number of candidates generated at each scan is reported in Figure 4. Figure 4 also reports the number of candidates for databases of sequences having length 24, 26 and 28. For the base of sequences having length 28 , the memory was exceeded and the process could not succeed. We made the same observation for PrefixSpan ${ }^{2}[18]$ where the number of intermediate sequences was similar to that of PSP with the same mere databases. If this phenomenon is not blocking for methods extracting the whole exact result (one can select the appropriate method depending on the dataset), the integration of such a method in our process for extracting dense periods is impossible because the worst case can appear in any batch ${ }^{3}$.

\section{$5 \quad$ Extracting Dense Periods}

In this section, we describe the steps allowing to obtain the dense periods of a Web access log. We also describe the neighborhood operators designed for PERIO, the heuristic presented in this paper.

\footnotetext{
${ }^{2}$ http://www-sal.cs.uiuc.edu/ hanj/software/prefixspan.htm

${ }^{3}$ In a web usage pattern, numerous repetitions of requests for pdf or php files, for instance, are usual.
} 


\section{$5.1 \quad$ Preprocessing}

This section is not intended to give details about the general preprocessing methods that can be applied to a Web access log. We assume that a preprocessing method such as [21] (identifying navigations, sessions, and robots) has already been applied to the log. The preprocessing described here is straight forward. It is described by figure 1. For each client, we extract the date of arrival and departure in the log. Then, the different dates are stored as "date, action, client". So, for each date we know if it corresponds to an arrival or a departure (thanks to the "action" field) and the client related to this action. Those records are then sorted by date in order to have an history of the log. Reading this history allows to know the next action to be performed for each date recorded (adding or deleting a client sequence in main memory).

\subsection{Heuristic}

Since our proposal is a heuristic-based miner, our goal is to provide a result having the following characteristics:

For each period $p$ in the history of the log, let realResult be the set of frequent behavioural patterns embedded in the navigation sequences of the users belonging to $p$. realResult is the result to obtain (i.e. the result that would be exhibited by a sequential pattern mining algorithm which would explore the whole set of solutions by working on the clients of $C_{p}$ ). Let us now consider perioResult the result obtained by running the method presented in this paper. We want to minimize $\sum_{i=0}^{\text {size(perioResult) }} S_{i} / S_{i} \notin$ realResult (with $S_{i}$ standing for a frequent sequence in perioResult), as well as maximize $\sum_{i=0}^{\text {size (realResult) }} R_{i} / R_{i} \in$ perioResult (with $R_{i}$ standing for a frequent sequence in realResult). In other words, we want to find most of the sequences occurring in realResult while preventing the proposed result becoming larger than it should (otherwise the set of all client navigations would be considered as a good solution, which is obviously wrong).

This heuristic is inspired from genetic algorithms and their neighborhood operators. Those operators are provided with properties of frequent sequential patterns in order to produce optimal candidates. The main idea of the Perio algorithm is to scan $P_{\text {stable }}$ the set of stable periods and, for each $p$ in $P_{\text {stable }}$ to propose candidates population thanks to previous frequent patterns and neighborhood operators. These candidates are then compared to the sequences of $C_{p}$ in order to know their threshold (or at least their distance from a frequent sequence). These two phases (neighborhood operators and candidate valuation) are explained in this section.

\subsubsection{Neighborhood Operators}

The neighborhood operators we used were validated by experiments performed on the Web logs of Inria Sophia Antipolis (see section 6). We chose "Genetic-like" operators as well as operators based on sequential pattern properties. We present here some of the most efficient operators for the problem presented in this paper. When we talk about random sequence, we use a biased random such that sequences having a high threshold may be chosen before sequences having a low threshold.

Finally, we evaluated the success rates for each of our operators thanks to the average number of frequent sequences compared to the proposed candidates. An operator having a success rate of $20 \%$ is an operator for which $20 \%$ of the proposed candidates are detected has frequent.

New frequent items: When a new frequent item occurs (after being requested by one or more users) it is used to generate all possible 2-candidate sequences with other frequent items. The candidate set generated is thus added to the global candidate set. Due to the number of candidate sequences to test, this operator only has a $15 \%$ ratio of accepted (i.e. frequent) sequences. This operator however remains essential since the frequent 2-sequences obtained are essential for other operators. 


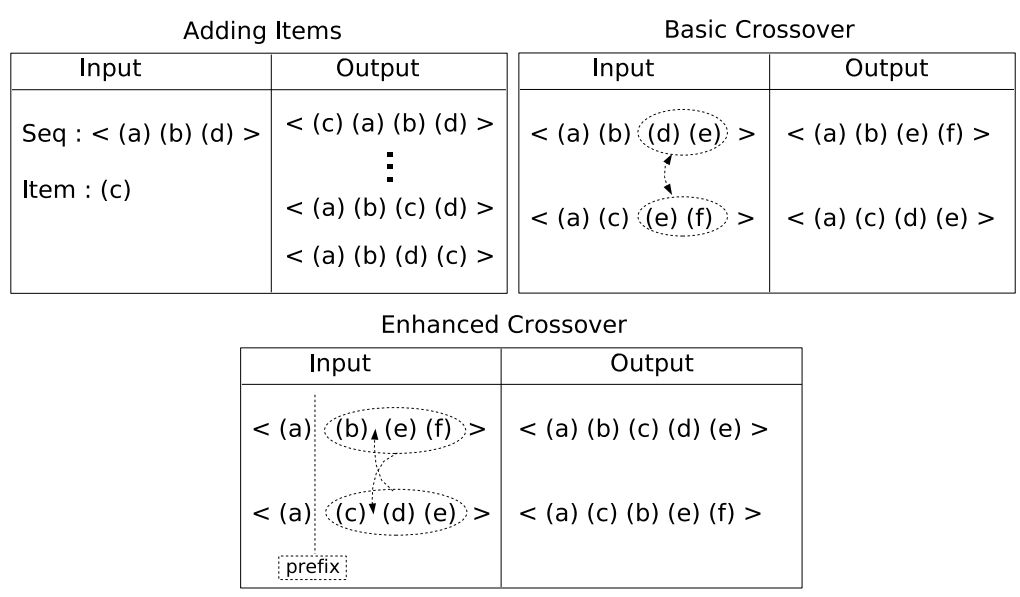

Figure 5: Some operators designed for extracting frequent navigation patterns

Adding items: This operator aims at choosing a random item among frequent items and adding this item to a random sequence $s$, after each item in $s$. This operator generates length $(s)+1$ candidate sequences. For instance, with the sequence $\langle$ (a) (b) (d) $>$ and the frequent item $c$, we will generate the candidate sequences $<$ (c) (a) (b) (d) $>$, $<$ (a) (c) (b) (d) $>$, $<$ (a) (b) (c) (d) $>$ and finally $<$ (a) (b) (d) (c) $>$. This operator has a $20 \%$ ratio of accepted sequences, but the sequences found are necessary for the following operators.

Basic crossover: This operator (largely inspired by genetic algorithms operators) uses two different random sequences and proposes two new candidates coming from their amalgamation. For instance, with the sequences $<$ (a) (b) (d) (e) $>$ and $<$ (a) (c) (e) (f) $>$, we propose the candidates $<$ (a) (b) (e) (f) $>$ and $<$ (a) (c) (d) (e) $>$. This operator has a good ratio $(50 \%)$ thanks to frequent sequences embedded in the candidates generated by previous operators.

Enhanced crossover: Encouraged by the result obtained when running the previous operator, we developed a new operator, designed to be an enhancement of the basic crossover, and based on the frequent sequences properties. This operator aims at choosing two random sequences, and the crossover is not performed in the middle of each sequence, but at the end of the longest prefix common to the considered sequences. Let us consider two sequences $<$ (a) (b) (e) (f) $>$ and $<$ (a) (c) (d) (e) $>$ coming from the previous crossover operator. The longest prefix common to these two sequences is $<$ (a) $>$. The crossover therefore starts after the item following $a$, for each sequence. In our example, the two resulting candidate sequences are, $<$ (a) (b) (c) (d) (e) $>$ and $<$ (a) (c) (b) (e) (f) $>$. This operator has a success ratio of $35 \%$.

Final crossover: An ultimate crossover operator was designed in order to improve the previous ones. This operator is based on the same principle as the enhanced crossover operator, but the second sequence is not randomly chosen. Indeed, the second sequence is chosen as being the one having the longest common prefix with the first one. This operator has a ratio of $30 \%$.

Sequence extension: This operator is based on the following idea: frequent sequences are extended with new pages requested. The basic idea aims at adding new frequent items at the end of several random frequent sequences. This operator has a success ratio of $60 \%$.

Figure 5 gives an illustration of some operators described in this section. 


\subsubsection{Candidate Evaluation}

The PERIO heuristic is described by the following algorithm:

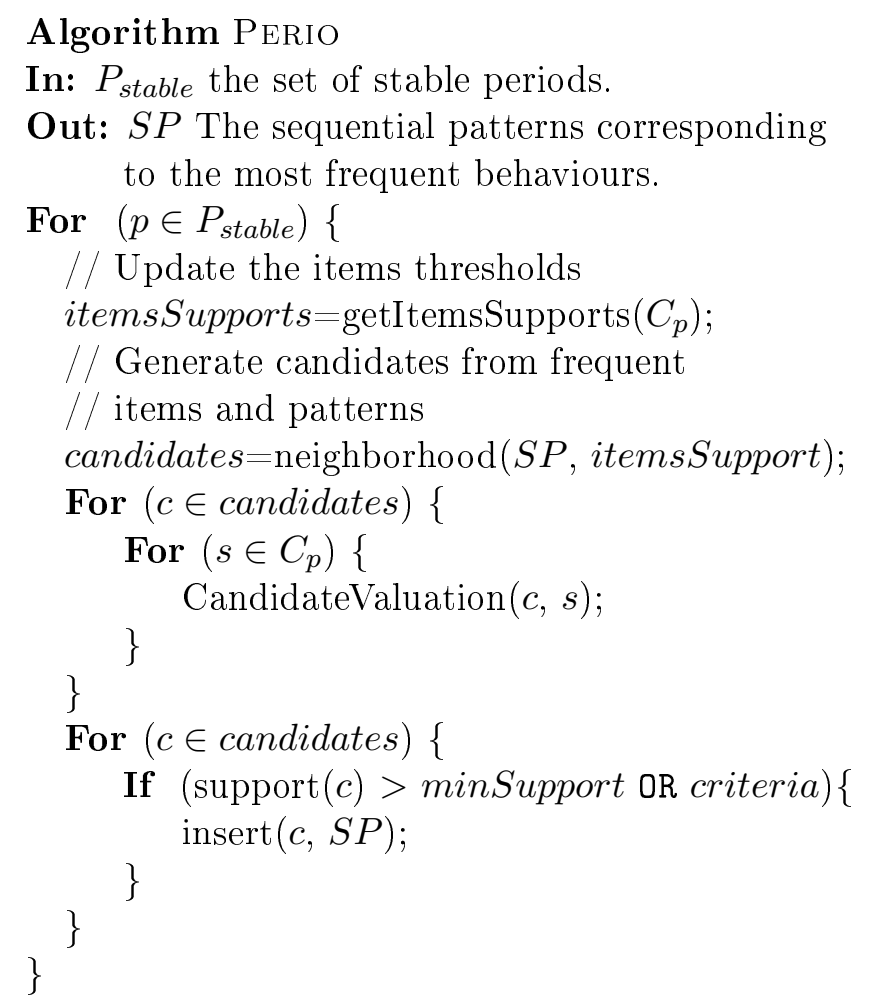

\section{End algorithm PERIO}

\section{Algorithm CandidateEvaluation}

In: $c$ a candidate to evaluate and $s$ the navigation sequence of the client.

Out: $p[c]$ the percentage given to $c$.

$/ /$ If $c$ is included in $s, c$ is rewarded

If $(c \subseteq s) p[c]=100+$ length $(c)$;

$/ /$ If $c$, having length 2 , is not included then

$/ /$ give $c$ the lowest mark.

If $($ lengthc) $\leq 2) p[c]=0$;

$/ /$ Else, give $s$ a mark and give

// largest distances a penalty

$p[c]=\frac{\text { length }(L C S(c, s)) * 100}{\text { length }(c)}-$ length $(c)$;

\section{End algorithm CANDIDATEEVALUATION}

For each stable period of $P_{\text {stable }}$, PERIO will generate new candidates and then compare each candidate to the sequence of $C_{p}$. The comparison aims at returning a percentage, representing the distance between the candidate and the navigation sequence. If the candidate is included in the sequence, the percentage should be $100 \%$ and this percentage decreases when the amount of interferences (differences between the candidate and the navigation sequence) increases. To evaluate this distance, the percentage is obtained by the fraction of the length of the longest common subsequence (LCS) [6] between $s$ and $c$, on the length of $s:|L C S(s, c)| /|s|$. Furthermore, in order to obtain frequent sequences that are as long as possible, we use an algorithm that rewards long sequences if they are included in the navigation sequence. On the other hand, the algorithm has to avoid long, not included, sequences (in order for the clients not to give a good mark to any long sequence). To cover all these parameters, the 
calculation performed by the client machine is described in the algorithm CANDIDATEEvaluation. Finally evaluated candidates having either their support greater than or equal to the minimal support value or corresponding to a "natural selective criteria" are stored into $S P$. This last criteria, which is user-defined, is a threshold corresponding to the distance between the candidate support and the minimal support. In our case, this criteria is used in order to avoid than the PERIOD heuristic leads towards a local optima.

\subsection{Result Summary and Visualization}

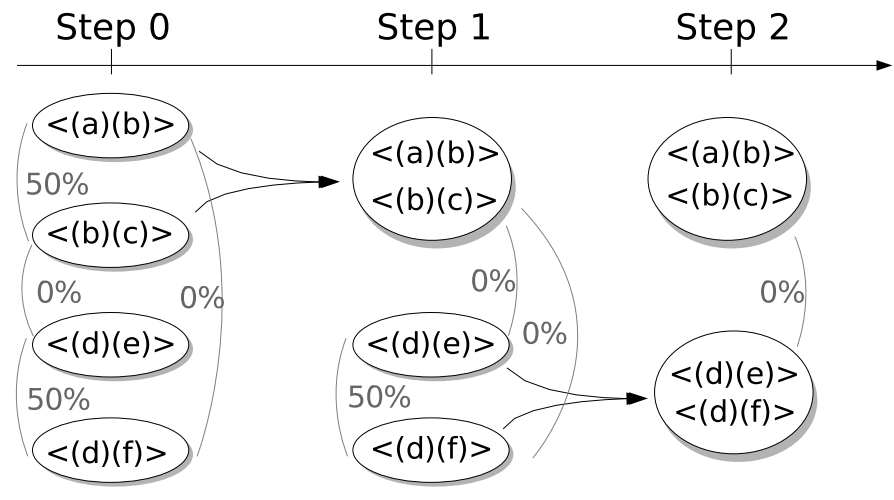

Figure 6: Clustering of sequential patterns before their alignment

Due to the number of candidates proposed by such a heuristic, the number of resulting sequences is very large. For instance, if the patterns $\langle(\mathrm{a})(\mathrm{b})>$ and $\langle(\mathrm{a})(\mathrm{b})(\mathrm{c})>$ are extracted by PERIO, then they will be both inserted in the result. In fact this problem cannot be reduced to the inclusion problem. As the size of extracted patterns is very long and as the delay of processing period has to be as short as possible, we could obtain patterns which are very close. Furthermore, extracted patterns could be very different since they represent different kind of behaviours. In order to facilitate the visualization of the issued result, we propose to extend the work of [11].

Our method performs as follows. We cluster together similar sequences. This operation is based on a hierarchical clustering algorithm [8] where the similarity is defined as follows:

\begin{tabular}{lllll} 
Step 1: & & & & \\
$S_{1}:$ & $<(\mathrm{a}, \mathrm{c})$ & $(\mathrm{e})$ & () & $(\mathrm{m}, \mathrm{n})>$ \\
$S_{2}:$ & $<(\mathrm{a}, \mathrm{d})$ & $(\mathrm{e})$ & $(\mathrm{h})$ & $(\mathrm{m}, \mathrm{n})>$ \\
\hline$S A_{12}:$ & $(\mathrm{a}: 2, \mathrm{c}: 1, \mathrm{~d}: 1): 2$ & $(\mathrm{e}: 2): 2$ & $(\mathrm{~h}: 1): 1$ & $(\mathrm{~m}: 2, \mathrm{n}: 2): 2$ \\
& & & & \\
Step 2: & & & & \\
$S A_{12}:$ & $(\mathrm{a}: 2, \mathrm{c}: 1, \mathrm{~d}: 1): 2$ & $(\mathrm{e}: 2): 2$ & $(\mathrm{~h}: 1): 1$ & $(\mathrm{~m}: 2, \mathrm{n}: 2): 2$ \\
$S_{3}:$ & $<(\mathrm{a}, \mathrm{b})$ & $(\mathrm{e})$ & $(\mathrm{i}, \mathrm{j})$ & $(\mathrm{m})>$ \\
\hline$S A_{13}:$ & $(\mathrm{a}: 3, \mathrm{~b}: 1, \mathrm{c}: 1, \mathrm{~d}: 1): 3$ & $(\mathrm{e}: 3): 3$ & $(\mathrm{~h}: 1, \mathrm{i}: 1, \mathrm{j}: 1): 2$ & $(\mathrm{~m}: 3, \mathrm{n}: 2): 3$ \\
& & & & \\
Step 3: & & & & \\
$S A_{13}:$ & $(\mathrm{a}: 3, \mathrm{~b}: 1, \mathrm{c}: 1, \mathrm{~d}: 1): 3$ & $(\mathrm{e}: 3): 3$ & $(\mathrm{~h}: 1, \mathrm{i}: 1, \mathrm{j}: 1): 2$ & $(\mathrm{~m}: 3, \mathrm{n}: 2): 3$ \\
$S_{4}:$ & $<(\mathrm{b})$ & $(\mathrm{e})$ & $(\mathrm{h}, \mathrm{i})$ & $(\mathrm{m})>$ \\
\hline$S A_{14}:$ & $(\mathrm{a}: 3, \mathrm{~b}: 2, \mathrm{c}: 1, \mathrm{~d}: 1): 4$ & $(\mathrm{e}: 4): 4$ & $(\mathrm{~h}: 2, \mathrm{i}: 2, \mathrm{j}: 1): 3$ & $(\mathrm{~m}: 4, \mathrm{n}: 2): 4$
\end{tabular}

Figure 7: Different steps of the alignment method with the sequences from example 2 

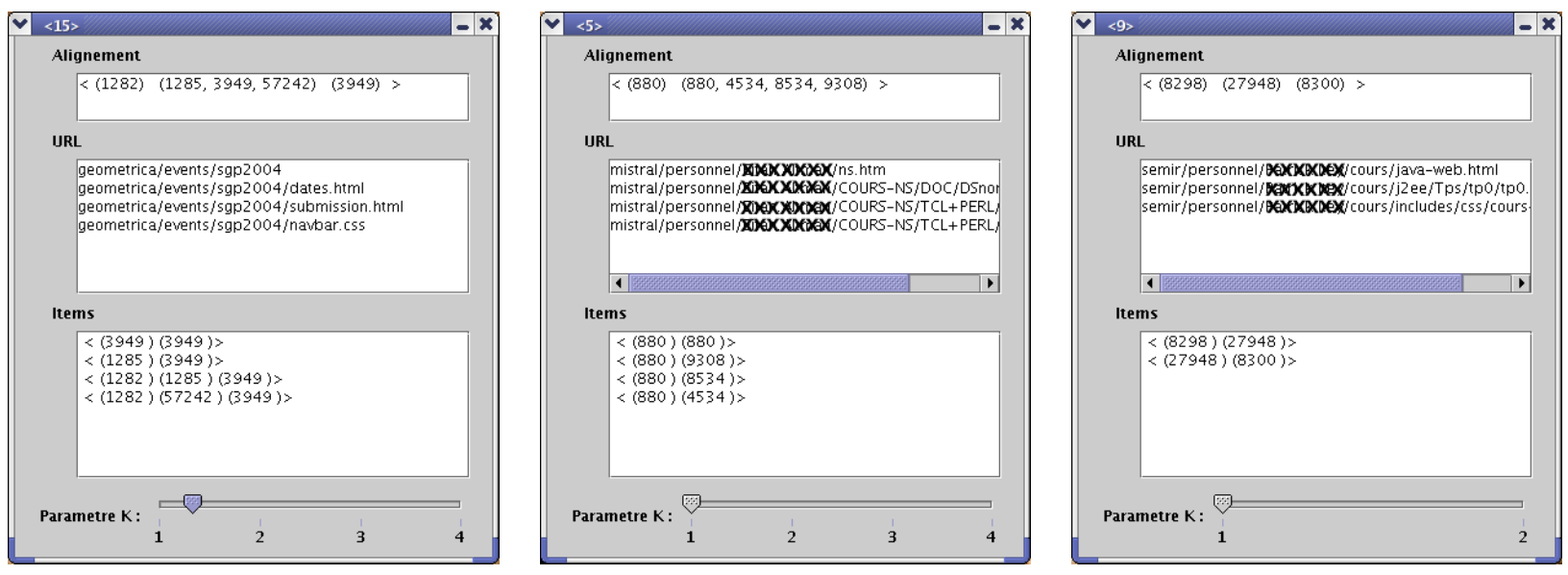

Figure 8: Screenshots of 3 clusters and their alignments.

Definition 9 Let $s_{1}$ and $s_{2}$ be two sequences.

Let $\left|L C S\left(s_{1}, s_{2}\right)\right|$ be the size of the longest common subsequence between $s_{1}$ and $s_{2}$. The degree of similarity between $s_{1}$ and $s_{2}$ is defined as: $d=\frac{2 \times\left|L C S\left(s_{1}, s_{2}\right)\right|}{\left|s_{1}\right|+\left|s_{2}\right|}$.

The clustering algorithm performs as follows. Each sequential pattern is first considered as a cluster (C.f. Step 0, Figure 6). At each step the matrix of similarities between clusters is processed. For instance, sequences $\langle(\mathrm{a})(\mathrm{b})>$ and $\langle(\mathrm{b})(\mathrm{c})>$ are similar at $50 \%$ since they share the same itemset (b). If we now consider the two following sequences $\langle(a)(b)\rangle$ and $\langle(d)(e)>$, their similarity is $0 \%$. The two close clusters are either $\{\langle(\mathrm{a})(\mathrm{b})\rangle,\langle(\mathrm{b})(\mathrm{c})\rangle\}$ or $\{\langle(\mathrm{d})(\mathrm{e})>,\langle(\mathrm{d})(\mathrm{f})\rangle\}$ since they have a same distance. They are grouped together into a unique cluster. Step "2" of Figure 6 shows the three clusters: $\{<(\mathrm{a})(\mathrm{b})>,<(\mathrm{b})(\mathrm{c})>\},\{<(\mathrm{d})(\mathrm{e})>\}$ and $\{<(\mathrm{d})(\mathrm{f})>\}$. This process is repeated until there are no more clusters having a similarity greater than 0 with an existing cluster. The last step of Figure 6 gives the result of the clustering phase: $\{<(\mathrm{a})(\mathrm{b})\rangle,\langle(\mathrm{b})(\mathrm{c})\rangle\}$ et $\{\langle(\mathrm{d})(\mathrm{e})\rangle,\langle(\mathrm{d})(\mathrm{f})\rangle\}$.

The clustering algorithm ends with clusters of similar sequences, which is a key element for sequences alignment. The alignment of sequences leads to a weighted sequence (as defined in [11]) represented as follows: $S A=<I_{1}: n_{1}, I_{2}: n_{2}, \ldots, I_{r}, n_{r}>: m$. In this representation, $m$ stands for the total number of sequences involved in the alignment. $I_{p}(1 \leq p \leq r)$ is an itemset represented as $\left(x_{i_{1}}: m_{i_{1}}, \ldots x_{i_{t}}: m_{i_{t}}\right)$, where $m_{i_{t}}$ is the number of sequences containing the item $x_{i}$ at the $n_{p}^{\text {th }}$ position in the aligned sequences. Finally, $n_{p}$ is the number of occurrences of itemset $I_{p}$ in the alignment. Example 2 describes the alignment process on 4 sequences. Starting from two sequences, the alignment begins with the insertion of empty items (at the beginning, the end or inside the sequence) until both sequences contain the same number of itemsets.

Example 2 Let us consider the following sequences:

$S_{1}=<(a, c)(e)(m, n)>, S_{2}=<(a, d)(e)(h)(m, n)>, S_{3}=<(a, b)(e)(i, j)(m)>, S_{4}=<(b)(e)$ $(h, i)(m)>$. The steps leading to the alignment of those sequences are detailed in Figure $\%$. First, an empty itemset is inserted in $S_{1}$. Then $S_{1}$ and $S_{2}$ are aligned in order to provide $S A_{12}$. The alignment process is then applied to $S A_{12}$ and $S_{3}$. The alignment method goes on processing two sequences at each step.

At the end of the alignment process, the aligned sequence ( $S A_{14}$ in Figure 7$)$ is a summary of the corresponding cluster. The approximate sequential pattern can be obtained by specifying $k$ : the number of occurrences of an item in order for it to be displayed. For instance, with the sequence $S A_{14}$ from Figure 7 and $k=2$, the filtered aligned sequence will be: $\langle(\mathrm{a}, \mathrm{b})(\mathrm{e})(\mathrm{h}, \mathrm{i})(\mathrm{m}, \mathrm{n})>$ (corresponding to items having a number of occurrences greater than or equal to $k$ ). 
Figure 8 illustrates three screenshots of the implemented visualization module. We can notice sequences (bottom part of the window) with the alignment performed on these sequences (top part of the window). Corresponding urls are described in the middle part. The left window illustrates behaviours corresponding to a SGP2004 Conference (Symposium on Geometry Processing) which was organized by the Geometrica team of Inria Sophia Antipolis. The meaning of the alignment is the following: users first access to the "home page" of the conference, then to the "Important Dates" or "Submission Page" (the navigation toolbar css, item (3949), could appear everywhere in this behaviour). Second and third windows illustrates behaviours on teacher pages. For instance, in the last windows, we can notice than two frequent behaviours were grouped together. These behaviours are then aligned in order to provide the end user with a more global behaviour.

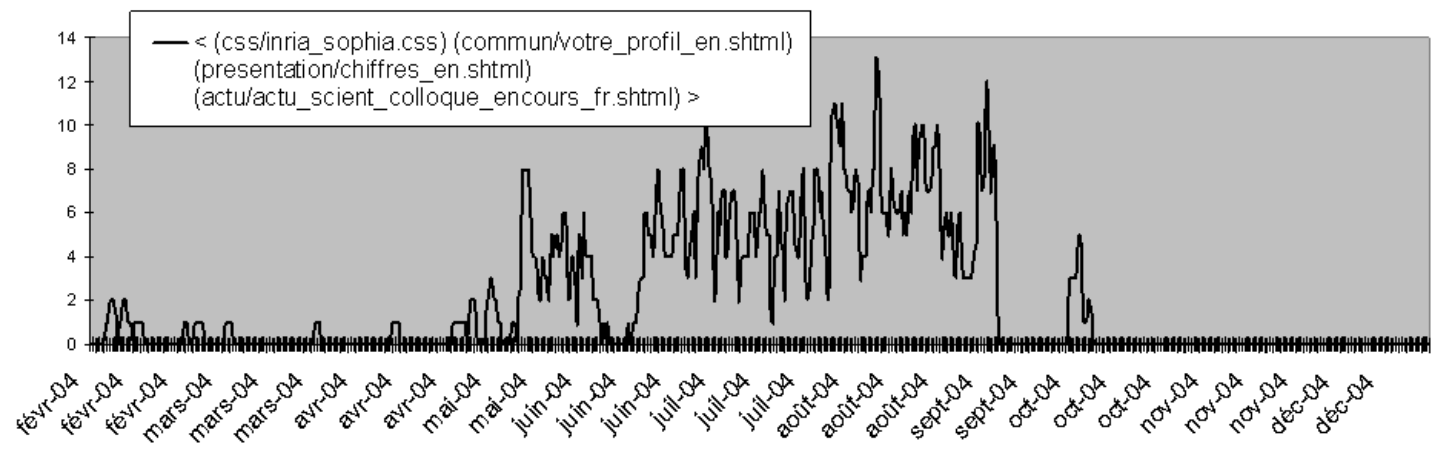

Figure 9: Un comportement fréquent sur plusieurs semaines consécutives

\section{Experiments}

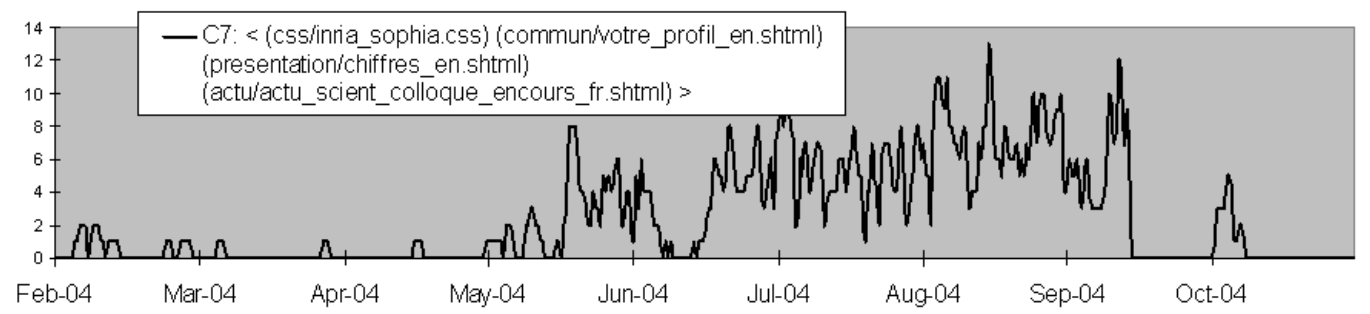

Figure 10: Peaks of frequency for a behaviour on a long period

PERIO was written in $\mathrm{C}++$ and compiled using gcc without any optimizations flags. All the experiments were performed on a PC computer with Pentium 2,1 Ghz running Linux (RedHat). They were applied on Inria Sophia Antipolis logs. These logs are daily obtained. At the end of a month, all daily log are merged together in a monthly log. During experiments we worked on 14 monthly logs. They were merged together in order to be provided with a unique log for a 14 months period (from January 2004 to March 2005). Its size is 14 Go of records. There are 3.5 millions of sequences (users), the average length of these sequences is 2.68 and the maximal size is 174 requests.

\subsection{Extracted Behaviours}

We report here some of the extracted behaviours. Those behaviours show that an analysis based on multiple division of the $\log$ (as described in this paper) allows obtaining behavioural patterns 

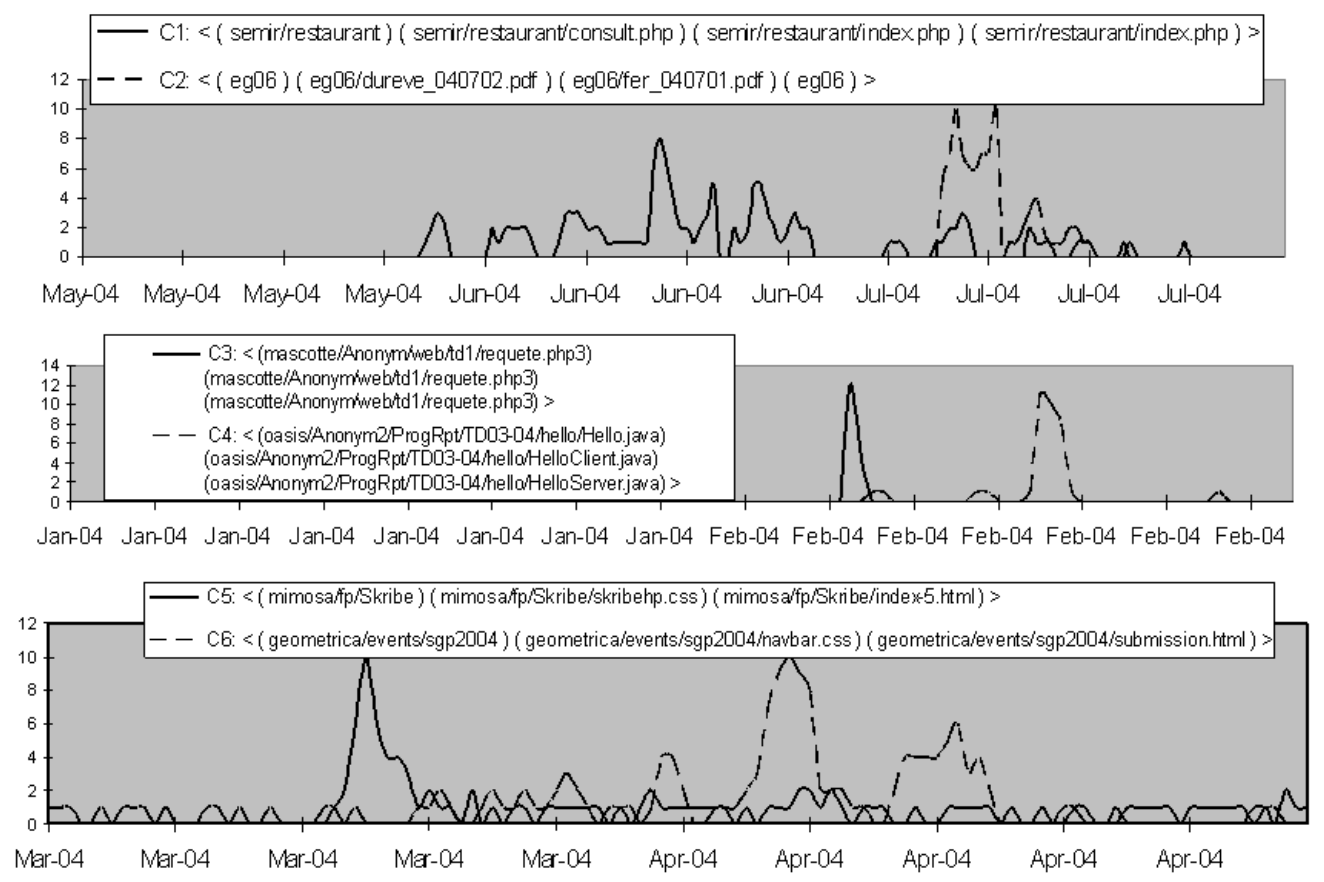

Figure 11: Peaks of frequency for $C 1, C 2, C 3, C 4, C 5$ and $C 6$

embedded in short or long periods. Execution time of PERIO on this log with a minimal support value of $2 \%$ is nearly 6 hours. The support of $2 \%$ was the best setting for obtaining interesting patterns and limiting the size of the output. We have found 1981 frequent behaviours which were grouped together on 400 clusters with techniques described in Section 5.3.

Figure 11 focuses on the evolution of the following behaviours:

- $C 1=<$ (semir/restaurant) (semir/restaurant/consult.php) (semir/restaurant/index.php) (semir/restaurant/index.php)>

- $C 2=<($ eg06) (eg06/dureve_040702.pdf) (eg06/fer_040701.pdf) (eg06)>

- $C 3=<$ (requete.php3) (requete.php3) (requete.php3)>

- $C 4=<$ (Hello.java) (HelloClient.java) (HelloServer.java) $>$

- $C 5=<(\operatorname{mimosa} / \mathrm{fp} /$ Skribe) $(\operatorname{mimosa} / \mathrm{fp} /$ Skribe/skribehp.css $)$ (mimosa/fp/Skribe/index-5.html) $>$

- $C 6=<(\operatorname{sgp} 2004)$ (navbar.css) (submission.html) $>$

All itemsets of behaviour $C 4$ are prefixed by "oasis/anonym2/Prog Rpt/TD03-04/hello/". For $C 3$ the prefix is "mascotte/ anonym3/web/td1/" and for C6 the prefix is "geometrica/events/".

The first behaviour $(C 1)$ corresponds to a typically periodic behaviour. Actually, the Inria's restaurant has been closed for a few weeks and people had to order a cold meal through a dedicated web site. This web site was located at "semir/restaurant". $C 2$ is representative of behaviours related to the recent "general assembly" of French researchers, hosted in Grenoble (France, Oct 2004).

Behaviours $C 3$ and $C 4$ correspond to navigation performed by students on pages about computer science courses and stored on some Inria researcher pages. 
When we have noticed the $C 5$ behaviours, we asked the reasons of such behaviours to the pages owner. His interpretation is that such behaviours are due to the large number of exchanged mails on March 2004 through the mailing list of Skribe (generating numerous navigations on the web pages of this project). Two different peaks appear, (begin of April and middle of April) for the behaviour $C 6$. Those peaks correspond in fact to the submission steps (respectively abstract and full papers) of articles for the SGP2004 Conference.

Some of the extracted behaviours do not occur on short periods only. Their occurrences are frequent on several weeks or even several months. Their support on the global log is related to the number of customers connected for each period. This is the case, for instance, of:

- $C 7=<($ css/inria_sophia.css) (commun/votre_profil_en.shtml) (presentation/chiffres_en.shtml) (actu/actu_scient_colloque_encours_fr.shtml) $>$

The evolution of $C 7$ is reported in Figure 10. We can observe that this behaviour occurs for 5 consecutive months (from May to September).

\subsection{Comparison to Sequential Pattern Mining}

Section 6.1 is devoted to showing some extracted behaviours and their content. In this section we aim at showing a comparison between our method on the one hand, and traditional method for sequential patterns on the other hand. We will show that the behaviours obtained by PERIO have such a low support that:

1. They cannot be extracted by a traditional sequential pattern mining algorithm.

2. The period they belong to cannot be identified by a traditional sequential pattern mining algorithm.

\begin{tabular}{|c|c|c|c|c|c|c|c|c|c|c|}
\hline & Max & Global & \% Global & PSP $_{\text {Global }}$ & Month & \% Month & PSP $_{\text {Month }}$ & Day & \% Day & PSP $_{\text {Day }}$ \\
\hline $\mathrm{C}_{1}$ & 13 & 507 & $0.0197 \%$ & - & $08-2004$ & $0.031 \%$ & - & Aug-09 & $0.095 \%$ & $20 \mathrm{~s}$ \\
\hline $\mathrm{C}_{2}$ & 8 & 69 & $0.0027 \%$ & - & $07-2004$ & $0.004 \%$ & - & Jun-10 & $0.2 \%$ & - \\
\hline $\mathrm{C}_{3}$ & 10 & 59 & $0.0023 \%$ & - & $07-2004$ & $0.004 \%$ & - & Jul-02 & $0.33 \%$ & $10 \mathrm{~s}$ \\
\hline $\mathrm{C}_{4}$ & 12 & 19 & $0.0007 \%$ & - & $02-2004$ & $0.006 \%$ & - & Feb-06 & $0.35 \%$ & $18 \mathrm{~s}$ \\
\hline $\mathrm{C}_{5}$ & 10 & 32 & $0.0012 \%$ & - & $02-2004$ & $0.01 \%$ & - & Feb-16 & $0.33 \%$ & $21 \mathrm{~s}$ \\
\hline $\mathrm{C}_{6}$ & 10 & 935 & 0.0364 & - & $02-2004$ & $0.09 \%$ & - & Mar -15 & $0.35 \%$ & $12 \mathrm{~s}$ \\
\hline $\mathrm{C}_{7}$ & 10 & 226 & $0.0088 \%$ & - & $04-2004$ & $0.01 \%$ & - & Apr-03 & $0.23 \mathrm{~s}$ & $8 \mathrm{~s}$ \\
\hline
\end{tabular}

Figure 12: Supports of the extracted behaviours at 3 granularities (Global, Month \& Day)

In Figure 12 we report several information about the behaviours presented in section 6.1. The meaning of each value is given in Figure 13. We give those information at three granularities (year, month and day). First of all, we give the maximum number of simultaneous occurrences of each behaviour in a stable period (column "Max"). Then we report the global support of this behaviour: the number of sequences containing the behaviour in the whole log file is given in column "Global" whereas the ratio is given in column "\% Global".

A first comparison is given with PSP on the whole log file for each behaviour. We report in $\mathrm{PSP}_{\text {Global }}$ the execution time of PSP on the whole log file with a support of $\%_{\text {Global }}$. We can observe that for each behaviour, PSP is unable to extract the patterns corresponding to the given support. The main reason is that this support is much lower than any traditional method for mining sequential patterns would accept. The number of frequent items for $C 6$ with a support of $0.0364 \%$ (bold "-") is 935 . In this case, the number of candidates having length 2 is $1,311,805$ so the main memory was rapidly 


\begin{tabular}{|l|l|}
\hline Max & The maximum number of simultaneous occurrences of this behaviour in a stable period \\
\hline Global & The support (total number of occurrences) of this behaviour in the global (14 months) log file \\
\hline$\%_{\text {Global }}$ & The support (percentage) corresponding to Global w.r.t the number of data sequences in the global log file \\
\hline PSP \\
Global & The execution time of PSP on the global log file with a minimum support of \% Global in a stable period \\
\hline Month & The month having the highest number of simultaneous occurrences of this behaviour in \\
\hline$\%_{\text {Month }}$ & The support (percentage) of this behaviour on Month \\
\hline PSP Month $_{\text { }}$ & The execution time of PSP on the log file corresponding to Month with a minimum support of $\%_{\text {Month }}$ \\
\hline Day & The day having the highest number of simultaneous occurrences of this behaviour in a stable period \\
\hline$\%_{\text {Day }}$ & The support (percentage) of this behaviour on Day \\
\hline PSP $_{\text {Day }}$ & The execution time of PSP on the log file corresponding to Day with a minimum support of $\%_{\text {Day }}$ \\
\hline
\end{tabular}

Figure 13: Legend for the table of Figure 12

overloaded and PSP could not succeed.

We also identified (by comparing months between each others) for each behaviour the month having the highest number of simultaneous occurrences of this behaviour in a stable period. In fact, the column "Month" corresponds to the month where this behaviour has the best support compared to other months. We report in column \% Month the support of each behaviour on the corresponding month

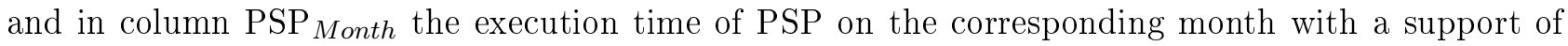
$\%$ Month. We can observe that PSP is unable to extract the sequential patterns corresponding to each month.

Finally, we identified for each behaviour the day having the highest number of simultaneous occurrences of this behaviour in a stable period (column "Day"). We report in column \% Day the support of each behaviour on the corresponding day and in column PSP Day the execution time of PSP on the corresponding day with a support of \% Day. We can observe that, at this granularity, PSP is able to extract most of the behaviours. Furthermore, PSP is even so fast that it could be applied on each day of the $\log$ and the total time would be around 70 minutes (420 days and an average execution time of approximately 10 seconds per day). Nevertheless, we have to keep in mind that with such an approach:

1. Undiscovered periods will remain (for instance a period of two consecutive days or a period of one hour embedded in one of the considered days).

2. Undiscovered behaviours will remain (embedded in the undiscovered periods).

3. The method would be based on an arbitrary division of the data (why working on each day and not on each hour or each week or each half day?).

Finally, in order to avoid the drawbacks enumerated above, the only solution would be to work on each stable period and apply a traditional sequential pattern algorithm. However this would require several millions of calls to the mining algorithm and the total execution time would be around 20 days (3,500,000 periods and an average execution time of approximately 0.5 seconds per period). Furthermore (as stated in section 4.3) this solution is not satisfying because of the long repetitive sequences that may be embedded in the data.

\section{Conclusion}

The proposition developed in this paper has shown that considering a log at large, i.e. without any division according to different values of granularity like traditional approaches, could provide the end user with a new kind of knowledge cutting: periods where behaviours are particularly significant and distinct. In fact, our approach aims at rebuilding all the different periods the log is made up with. 
Nevertheless, by considering the log at large (several month, several years, ...) we have to deal with a large number of problems: too many periods, too low frequency of behaviours, inability of traditional algorithms to mine sequences on one of these periods, etc. We have shown that a heuristic-based approach is very useful in that context and by indexing the log, period by period, we can extract frequent behaviours if they exist. Those behaviours could be very limited on time, or frequently repeated but their main particularity is that they are very few on the logs and they are representative of a dense period. Conducted experiments have shown different kind of behaviours concerning for instance either students, conferences, or restaurants. These behaviours were completely hidden on the $\log$ files and cannot be extracted by traditional approaches since they are frequent on particular periods rather than frequent on the whole log.

\section{References}

[1] R. Agrawal, T. Imielinski, and A. Swami. Mining Association Rules between Sets of Items in Large Databases. In Proceedings of the 1993 ACM SIGMOD Conf., pages 207-216, Washington DC, USA, May 1993.

[2] R. Agrawal and R. Srikant. Mining Sequential Patterns. In Proceedings of the 11th Int. Conf. on Data Engineering (ICDE'95), Tapei, Taiwan, March 1995.

[3] F. Bonchi, F. Giannotti, C. Gozzi, G. Manco, M. Nanni, D. Pedreschi, C. Renso, and S. Ruggieri. Web log data warehousing and mining for intelligent web caching. Data Knowledge Engineering, 39(2):165-189, 2001.

[4] World Wide Web Consortium. httpd-log files. In http://lists.w3.org/Archives, 1998.

[5] Robert Cooley, Bamshad Mobasher, and Jaideep Srivastava. Data preparation for mining world wide web browsing patterns. Knowledge and Information Systems, 1(1):5-32, 1999.

[6] T. Cormen, C. Leiserson, and R. Rivest. Introduction to Algorithms. MIT Press.

[7] U.M. Fayad, G. Piatetsky-Shapiro, P. Smyth, and R. Uthurusamy, editors. Advances in Knowledge Discovery and Data Mining. AAAI Press, Menlo Park, CA, 1996.

[8] J. Han and M. Kamber. Data Mining, concepts and techniques. Morgan Kaufmann, 2001.

[9] B. Hay, G. Wets, and K. Vanhoof. Mining Navigation Patterns Using a Sequence Alignment Method. Knowl. Inf. Syst., 6(2):150-163, 2004.

[10] http Analyze. http://www.http-analyze.org/.

[11] H. Kum, J. Pei, W. Wang, and D. Duncan. ApproxMAP: Approximate mining of consensus sequential patterns. In Proceedings of SIAM Int. Conf. on Data Mining, San Francisco, CA, 2003.

[12] F. Masseglia, F. Cathala, and P. Poncelet. The PSP Approach for Mining Sequential Patterns. In Proceedings of the 2nd European Symposium on Principles of Data Mining and Knowledge Discovery (PKDD'98), pages 176-184, Nantes, France, September 1998.

[13] F. Masseglia, P. Poncelet, and R. Cicchetti. An efficient algorithm for web usage mining. Networking and Information Systems Journal (NIS), April 2000.

[14] N. Meger and C. Rigotti. Constraint-Based Mining of Episode Rules and Optimal Window Sizes. In Proc. of the 8th European Conference on Principles and Practice of Knowledge Discovery in Databases (PKDD), pages 313-324, Pisa, Italy, September 2004.

[15] B. Mobasher, H. Dai, T. Luo, and M. Nakagawa. Discovery and evaluation of aggregate usage profiles for web personalization. Data Mining and Knowledge Discovery, 6(1):61-82, January 2002.

[16] M. Nakagawa and B. Mobasher. Impact of Site Characteristics on Recommendation Models Based On Association Rules and Sequential Patterns. In Proceedings of the IJCAI'03 Workshop on Intelligent Techniques for Web Personalization, Acapulco, Mexico, August 2003.

[17] C. Neuss and J. Vromas. Applications CGI en Perl pour les Webmasters. Thomson Publishing, 1996.

[18] J. Pei, J. Han, B. Mortazavi-Asl, H. Pinto, Q. Chen, U. Dayal, and MC. Hsu. PrefixSpan: Mining Sequential Patterns Efficiently by Prefix-Projected Pattern Growth. In 17th International Conference on Data Engineering (ICDE), 2001.

[19] M. Spiliopoulou, L. C. Faulstich, and K. Winkler. A data miner analyzing the navigational behaviour of web users. In Proceedings of the Workshop on Machine Learning in User Modelling of the ACAI'99 Int. Conf., Creta, Greece, July 1999. 
[20] R. Srikant and R. Agrawal. Mining Sequential Patterns: Generalizations and Performance Improvements. In Proceedings of the 5th Int. Conf. on Extending Database Technology (EDBT'96), pages 3-17, Avignon, France, September 1996.

[21] D. Tanasa and B. Trousse. Advanced Data Preprocessing for Intersites Web Usage Mining. IEEE Intelligent Systems, 19(2):59-65, April 2004. ISSN 1094-7167.

[22] Webalizer. http://www.mrunix.net/webalizer/.

[23] J. Zhu, J. Hong, and J. G. Hughes. Using Markov Chains for Link Prediction in Adaptive Web Sites. In Proceedings of Soft-Ware 2002: First Int. Conf. on Computing in an Imperfect World, pages 60-73, Belfast, UK, April 2002. 